\title{
Tranexamic Acid Use in the Total Hip and Total Knee Arthroplasty Population at an Academic Medical Center: a Retrospective Review of Postoperative Outcomes
}

\author{
Brianne E. Kaufman, Pharm.D, ${ }^{1}$ Julie A. Murphy, Pharm.D., FASHP, FCCP, \\ BCPS, ${ }^{2}$ Natalie Tuttle, Pharm.D., BCPS, ${ }^{1}$ Daniel Gehling, MD $^{3}$ \\ ${ }^{I}$ Pharmacy Services, University of Toledo Medical Center, USA \\ ${ }^{2}$ Pharmacy Practice, University of Toledo College of Pharmacy and Pharmaceutical Sciences, USA \\ ${ }^{3}$ Orthopaedics, University of Toledo Medical Center, USA
}

\begin{abstract}
Purpose Excessive blood loss and subsequent blood transfusions in orthopedic procedures have been associated with considerable morbidity and mortality. The primary objective of this study was to determine the incidence of perioperative blood transfusion after hip or knee arthroplasty with the use of tranexamic acid (TXA) versus standard of care. Methods: Any adult patient who underwent a total hip or knee arthroplasty between October 1, 2012 and September 30, 2015 was eligible for inclusion. Patients received $1000 \mathrm{mg}$ of intravenous tranexamic acid preoperatively. Results: Two hundred ninety-eight patients were included, 148 in the TXA group, and 150 in the standard of care group. In the TXA group, 30 patients required a blood transfusion, versus 48 patients in the standard of care group ( $p=0.021)$. Conclusion: TXA was associated with a significantly lower need for blood transfusion and shorter median hospital length of stay without an increase in thromboembolic complications.
\end{abstract}

Keywords: arthroplasty, blood transfusion, thromboembolic complication, tranexamic acid

\section{Introduction}

Hip and knee arthroplasties are becoming increasingly more frequent procedures in the United States, with orthopedic cases of this type expected to double in the next 10 years ${ }^{[1]}$. One safety concern from these surgeries is the risk of bleeding. In fact, the cost of blood management for knee arthroplasty has increased over $80 \%$ from 1991 to $2008^{[2]}$. Many strategies to prevent the need for blood transfusion and limit the overall cost of these procedureshave been used. The use of tranexamic acid (TXA) in hip and knee arthroplasties has been studied to augment the need for blood transfusions, decrease hospital length of stay, and reduce overall cost.

TXA is a synthetic analog of the amino acid lysine. It providesan antifibrinolytic effect through competitive inhibition of the lysine binding site of plasminogen activation and, at higher concentrations, acts as a noncompetitive inhibitor of plasmin. It is often used for this antifibrinolytic effect in orthopedic surgeries such as total hip or knee arthroplastyin an effort to decrease intra- and postoperative bleeding. This use is not FDAapproved, as TXA is only FDA-approved for the treatment of hemophilia and menorrhagia. However, several hospitals have implemented protocols for the use of TXA in their orthopedic surgeries. Debate exists on the most effective route of administration for TXA in this setting, as oral, topical, intraarticular, and intravenous (IV) dosing strategies have been reported. Regardless of route, evidence to support the routine use of TXA in total hip or total knee arthroplasty has developed over the past decade.Utilizing retrospective patient chart review, we compared the characteristics and outcomes between patients receivingpreoperativeTXAwith those receiving standard of care to minimize bleeding, and subsequently analyzed whether the use of TXA was associated with reduced rates of blood transfusion. We hypothesized that the use of TXA reduced the need for blood transfusions without increasing postoperative thromboembolic complications.

\section{Methods}

The study was approved by the local ethics committee and was performed in accordance to the Declaration of Helsinki.This was a single center, retrospective cohortexamining the rates of blood transfusion in patients who received TXAversus standard of care alone perioperatively. Standard of care was defined as strategies to minimize bleeding, including routine hemostasis during surgery, regional anesthesia, etc. Patients who received TXA also received standard of care. The charts of patients 18 years of age or older who underwent total knee arthroplasty, total hip arthroplasty, knee revision, or hip revision between October 1, 2012 to September 30, 2015 at the academic medical centerwere reviewed. Patients were excluded if they had a hypersensitivity to TXA, religious objection to autologous blood transfusion, or were pregnant. The main intervention variable was the use ofTXA 1000 milligramsintravenouslygiven immediately prior to the initial incision, according to institution-approved policy; the policy went into effect in September 2013. 
The primary outcome was to determine the incidence of blood transfusion requirements in patients receiving TXA versus standard of care. The need for blood transfusion was documented via premade blood transfusion order sets that were scanned into the patient's medical record. Any blood transfusion that occurred intraoperatively or postoperatively was included.Secondary outcomes includedhospital length of stay, postoperative thromboembolic complications, hemoglobin levels, and 30-day readmission rates. Patient characteristics analyzed included age, sex, body mass index, and race (white, black, other). Comorbid conditions (diabetes mellitus, hypertension, history of myocardial infarction, history of gastrointestinal (GI) bleed, history of deep venous thromboembolism (DVT) or pulmonary embolism, heart failure, arrhythmias, cerebrovascular disease, chronic kidney disease, cancer, sickle cell anemia, cirrhosis, and hepatitis)were also collected using charted ICD-9 and ICD-10 codes. Preoperative and postoperative hemoglobin values were collected. Preoperative lab values were defined as being obtained within 45 days prior to surgery and postoperativelab values were defined as being obtained up to30 days post-surgery, with the recorded valuenearest to incision or closure time, respectively, used in statistical analysis. Procedure related variables included type of procedure (total hip or knee arthroplasty, hip revision, or knee revision), tourniquet time if applicable, length of surgery, use of TXA, theamount of estimated blood loss, and subsequent units of blood transfused. Postoperative variables measured included use of postoperative anticoagulants and the development of a thromboembolic complication during the surgery admission. All anticoagulants, besides aspirin at a dose of $81 \mathrm{mg}$ oraldaily, were considered acceptable forms of post-operative DVT prophylaxis ${ }^{[3]}$.

The data was analyzed using SPSS software (version 23). The association between TXA use and study variables was assessed using $\chi^{2}$ tests for categorical variables and t tests for continuous variables. Mann Whitney $\mathrm{U}$ was utilized for non-normally distributed variables. A difference of $20 \%$ between groups with $80 \%$ power was needed to show statistical significance, with 150 patients needed per treatment arm. Differences were considered significant if the $\mathrm{p}$-value was $\leq 0.05$.

\section{Results}

Two hundred ninety-eight patients were eligible for inclusion. One hundred forty-eight patients received TXA and 150 patients received the standard of careonly. Baseline characteristics were similar between the two groups, except heart failure was more prevalent in the TXA group (Table 1).

Table 1: Baseline Characteristics

\begin{tabular}{|c|c|c|c|}
\hline Characteristic & TXA (n=148) & Standard Procedure $(n=150)$ & $P$ value \\
\hline Male, n (\%) & $60(40.5)$ & $64(42.7)$ & 0.71 \\
\hline Age (years), mean \pm SD & $63.4 \pm 12.33$ & $62.4 \pm 12.45$ & 0.438 \\
\hline BMI $\left(\mathrm{kg} / \mathrm{m}^{2}\right)$, median $(\mathrm{IQR})$ & $33.6(27.3-41.7)$ & $32.7(27.5-39.15)$ & 0.556 \\
\hline Race, $\mathrm{n}(\%)$ & & & 0.340 \\
\hline White & $124(83.8)$ & $121(80.7)$ & \\
\hline Black & $22(14.7)$ & $21(14)$ & \\
\hline Other & $2(1.5)$ & $8(5.2)$ & \\
\hline \multicolumn{4}{|l|}{ Comorbidities, n (\%) } \\
\hline Hypertension & $105(71)$ & $109(73)$ & 0.741 \\
\hline Diabetes Mellitus & $52(35)$ & $47(31)$ & 0.486 \\
\hline Arrhythmias & $26(18)$ & $16(11)$ & 0.087 \\
\hline Heart Failure & $15(10)$ & $6(4)$ & 0.039 \\
\hline Cancer & $14(9)$ & $17(11)$ & 0.596 \\
\hline Chronic Kidney Disease & $14(9)$ & $15(10)$ & 0.875 \\
\hline Coronary Artery Disease & $8(5)$ & $10(7)$ & 0.648 \\
\hline History of thromboembolism & $6(4)$ & $8(5)$ & 0.602 \\
\hline Cerebral vascular disease & $5(3)$ & $9(6)$ & 0.285 \\
\hline Cirrhosis/Hepatitis & $3(2)$ & $5(3)$ & 0.723 \\
\hline History of GI bleed & $2(1)$ & $1(0.6)$ & 0.621 \\
\hline Sickle Cell Anemia & $1(0.6)$ & $0(0)$ & 0.497 \\
\hline
\end{tabular}

Primary and secondary outcomes are displayed in Table 2. Patients who received TXA had a lower rate of blood transfusion compared to those who received standard of care $(20 \%$ vs. $32 \%, \mathrm{p}=0.021)$; those who received TXA also had a shorter length of stay ( 3 days vs 4 days, $p=<0.001$ ). There was no difference in thromboembolic complications or 30-day readmission rates between those who received TXA versus those who received standard of care only. Both preoperative and postoperative hemoglobin values were available, in the prespecified timeframe, for only 136 patients in each group. There was no significant difference in change in hemoglobin between those receiving TXA versus standard of care. 
Tranexamic Acid Use in the Total Hip and Total Knee Arthroplasty Population at an Academic

Table 2: Primary and Secondary Outcomes

\begin{tabular}{|c|c|c|c|}
\hline \multicolumn{4}{|l|}{ Primary Outcome } \\
\hline & TXA $(n=148)$ & Standard Procure $(n=150)$ & P Value \\
\hline Blood Transfusion, $\mathrm{n}(\%)$ & $30(20)$ & $48(32)$ & 0.021 \\
\hline \multicolumn{4}{|l|}{ Secondary Outcomes } \\
\hline & TXA $(n=148)$ & Standard Procure $(n=150)$ & P Value \\
\hline Length of stay (days), median (IQR) & $3(3-5)$ & $4(3-6)$ & $<0.001$ \\
\hline Thromboembolic complication, n (\%) & $3(2)$ & $4(3)$ & 1.0 \\
\hline \multirow[t]{2}{*}{30 day readmission, $\mathrm{n}(\%)$} & $6(4)$ & $13(8.7)$ & 0.103 \\
\hline & TXA $(n=136)$ & Standard Procure $(n=136)$ & \\
\hline Difference in hemoglobin (mg/dL), median (IQR) & $-2.7(-3.48--1.03)$ & $-2.4(-4.2--1.7)$ & 0.088 \\
\hline
\end{tabular}

Table 3 compares surgery characteristics between groups. There was no difference in procedure type. The duration of surgery (136 minutes and 147 minutes, $\mathrm{p}=0.002)$ and total tourniquet time (100 minutes and 123 minutes, $\mathrm{p}=0.001$ ) was shorter in patients who received TXA versus stand of care, respectively. There was no difference in the recorded intraoperative blood loss or the amount of blood transfusedintra- and postoperatively between groups. There was also no difference in the types of anticoagulation used postoperatively between groups.

Table 3: Surgery Characteristics

\begin{tabular}{|c|c|c|c|}
\hline Characteristic & TXA $(n=148)$ & Standard Procedure $(n=150)$ & P value \\
\hline Procedure Type, n (\%) & & & 0.270 \\
\hline Knee Arthroplasty & $49(33.1)$ & $46(30.7)$ & \\
\hline Hip Arthroplasty & $41(27.7)$ & $35(23.3)$ & \\
\hline Knee Revision & $30(20.3)$ & $34(22.7)$ & \\
\hline Hip Revision & $28(18.9)$ & $35(23.3)$ & \\
\hline Surgery Duration (min), median (IQR) & $136(107-165)$ & $147(114.5-179.5)$ & 0.002 \\
\hline Blood Loss (ml), median (IQR) & $250(100-600)$ & $250(100-450)$ & 0.570 \\
\hline Use of postoperative anticoagulation, $\mathrm{n}(\%)$ & $144(97.3)$ & $149(99.3)$ & 0.212 \\
\hline Type of anticoagulation & & & 0.151 \\
\hline Enoxaparin & $131(89)$ & $145(97)$ & \\
\hline Aspirin & $17(11)$ & $5(3)$ & \\
\hline Warfarin & $11(7)$ & $14(9)$ & \\
\hline Heparin & $5(3)$ & $8(5)$ & \\
\hline New oral anticoagulant & $4(3)$ & $2(1)$ & \\
\hline \multirow[t]{2}{*}{ No anticoagulation } & $4(3)$ & $1(0.6)$ & \\
\hline & TXA $(n=59)$ & Standard Procedure $(n=67)$ & \\
\hline \multirow[t]{2}{*}{ Tourniquet Time (min), median (IQR) } & $100(83-124)$ & $123(95-140)$ & 0.001 \\
\hline & TXA $(n=30)$ & Standard Procedure $(n=48)$ & \\
\hline Transfusion Amount (units), median (IQR) & $2(1.0-2.0)$ & $2(2.0-2.0)$ & 0.342 \\
\hline
\end{tabular}

\section{Discussion}

In this retrospective cohort of 298 patients, TXA was found to be associated with a $12 \%$ absolute reduction in the need for allogenic or autologous blood transfusions, without an increase in thromboembolic complications, regardless of the type of post-operative anticoagulation used. One patient in the TXA group did expire, however this was not found to be related to surgery complications. TXA was also found to be associated with a decrease in length of stay, surgery duration, and tourniquet time.Two orthopedic surgeons perform these procedures at the academic medical center. When the TXA policy was approved in September 2013, one surgeon began to use TXAduring a majority of his procedures while the other surgeon continued to use the standard of care to help minimize blood loss. One strength of the study is the inclusion of patients before the above surgeon began using TXA in September 2013. This helps minimize confounding variables such as surgeon technique, etc.Another strength of this study was the inclusion of patients regardless of their past medical history. For the surgeon who uses TXA, he will only exclude patients from its use if they have a history of thromboembolism, however this is not an absolute contraindication. In the TXA group, only $4 \%$ of the patients had a history of a prior thromboembolism. Finally, the dosing regimen implemented at our institution (1000mg IV given prior to surgery) was similar to that previously published by Irwin and colleagues, who used $15 \mathrm{mg} / \mathrm{kg}$ IV (max $1200 \mathrm{mg}$ ) given at the induction of anesthesia ${ }^{[4]}$.

Our study has a fewlimitations. First, two different orthopedic surgeons performed the surgeries;although, there was an effort to minimize the potentialbias of surgeon technique by expanding the timeframe for the standard of care group, which included both surgeons. Secondly, due to the retrospective nature of the study, hemoglobin values were not available for some patients, therefore leading to exclusion of thesepatients from secondary outcome analysis. Transfusion triggers were also not well defined. They may be 
based on hemoglobin levels or may depend on surgeon specific criteria based on multiple variables. However, there was little, if any, difference in the overall practice between those who received TXA and those who did not. With regard to the time of TXA administration, the study was limited to the categorical ("yes or no") use of TXA. A majority of patients received TXA preoperatively, however 40 patients did receive the dose intraoperative due to unavailability of TXA immediately prior to the incision. Our study did not find a difference in venous thromboembolism (VTE) rate, however, with the event rate being so small, there may not have been a large enough sample size to find a difference. Finally, we only reported thromboembolic complications that occurred during the patient's surgery admission and did not follow after discharge. Nevertheless, a study that reviewed TKA and THA patients for six weeks, found that over $90 \%$ of all lifethreatening complications occurred within 4 days after surgery ${ }^{[5]}$.

Multiple studies, including randomized controlled trials ${ }^{[-9]}$ and meta-analyses ${ }^{[10-14]}$ have found the same promising results on the effectiveness of TXA. In accordance with our research, multiple other studies have been underpowered to detect a difference in VTE rates. One of the largest retrospective cohorts to date included over 11,000 patients who received TXA and found that neither the odds of VTE nor adjusted odds of death were significant with TXA administration ${ }^{[15]}$. Blood product administration is associated with various possible complications, including but not limited to, transfusion related acute lung injury, transfusion related reactions, infections, hemochromatosis, and volume overload ${ }^{[16]}$. In addition, blood transfusion can be a significant cost to the patient. Forbes and colleagues found the average acquisition cost to be $\$ 155$, however this only made up $37 \%$ of the cost; the other $63 \%$ consists of blood bank handling, laboratory tests, and blood administration ${ }^{[17]}$.One study reported a reductioninhospital cost of $\$ 879$ for orthopedic patients who receive TXA ${ }^{[18]}$.

\section{Conclusion}

Our retrospective cohort found that TXA was associated with a significantly reduced rate of blood transfusions without an increase in thromboembolic complications. In accordance with the results from previous studies showing efficacy and safety of TXA use in orthopedic patients, widespread use of TXA as standard of care may result in a decrease in need for blood transfusions therefore reducing potential complications and direct costs to hospitals and patients.

\section{Acknowledgements}

We would like to acknowledge Todd Gundrum, Pharm.D., BCPS, and Sheena Burwell, Pharm.D. for their assistance in preparing the research for institutional review board approval. We would also like to acknowledge Zachary Henz, Pharm.D. and John Macko, Pharm.D., MBA for their assistance in creating the research database.

\section{References}

[1] Iorio R, Davis CM, III, Healy WL et al, Impact of the economic downturn on adult reconstruction surgery,J Arthroplasty 25, 2010, 1005-1014.

[2] Rana AJ, Iorio R, Healy WL, Hospital economics of primary THA decreasing reimbursement and increasing cost, 1990 to 2008 , ClinOrthop469, 2008, 355-361.

[3] Falck-Ytter Y, Francis CW, Johanson NA, Curley C, Dahl OE, Schulman S et al, Prevention of VTE in orthopedic surgery patients: antithrombic therapy and prevention of thrombosis, $9^{\text {th }}$ ed: American College of Chest Physicians evidence-based clinical practice guidelines, Chest 141(suppl), 2012, 278S-325S.

[4] Irwin A, Khan SK, Jameson SS, Tate RC, Copeland C, Reed MR, Oral versus intravenous tranexamic acid in enhanced-recovery primary total hip and knee replacement: results of 3000 procedures,Bone Joint J 95, 2013, 1556-1561.

[5] Parvizi J, Mui A, Purtill JJ, Sharkey PF, Hozack WJ, Rothman RH, Total joint arthroplasty: when do fatal or near-fatal compliationsoccur?,J Bone Joint Surg Am 89, 2007, 780-785.

[6] Oremus K, Sostaric S, Trkulja V, Haspl M, Influence of tranexamic acid on postoperative autologous blood transfusion in primary total hip and knee arthroplasty: a randomized controlled trial,Transfusion 54, 2014, 31-41.

[7] Charoencholvanich K, Siriwattanasakul P, Tranexamic acid reduces blood loss and blood transfusion after TKA,ClinOrthopRelat Res 469, 2011, 2874-2880.

[8] Good L, Peterson E, Lisander B,Tranexamic acid decreases external blood loss but not hidden blood loss in total knee replacement, Br J Anaesth 90, 2003, 596-599.

[9] Jansen AJ, Andreica S, Claeys M, D'Haese J, Camu F, Jochmans K,Use of tranexamic acid for an effective blood conservation strategy after total knee arthroplasty,Br J Anaesth 83, 1999, 596-601.

[10] Wei Z, Liu M, The effectiveness and safety of tranexamic acid in total hip or knee arthroplasty: a meta-analysis of 2720 cases,Transfus Med 25, 2015, 151-162.

[11] Shemshaki H, Nourian SM, Nourian N, Dehghani M,Mokhtari M, Mazoochian F, One step closer to sparing total blood loss and transfusion rate in total knee arthroplasty: a meta-anaylsis of different methods of tranexamic acid administration,Arch Orthop Trauma Surg 135, 2015, 573-588.

[12] Huang F, Wu D, Ma G, Yin Z, Wang Q, The use of tranexamic acid to reduce blood loss and transfusion in major orthopedic surgery: a meta-analysis, J Surg Res 186, 2014, 318-327.

[13] Zhou XD, Tao LJ, Li J, Wu LD,Do we really need tranexamic in total hip arthroplasty? A meta-analysis of nineteen randomized controlled trials,Arch Orthop Trauma Surg 133, 2013, 1017-1027.

[14] Yang ZG, Chen WP, Wu LD,Effectiveness and safety of tranexamic acid in reducing blood loss in total knee arthroplasty: a metaanalysis, J Bone Joint Surg Am 94, 2012, 1153-1159. 
[15] Duncan CM, Gillette BP, Jacob AK, Sanchez-Sotelo J, Smith HM, Venous thromboembolism and mortality associated with tranexamic acid use during total hip and knew arthroplasty,J arthroplasty 30, 2015, 272-276.

[16] Vamvakas EC, Blajchman MA, Transfusion-related mortality:the ongoing risks of allogeneic blood transfusion and the available strategies for their prevention,Blood 113(15), 2009, 3406-17.

[17] Forbes JM, Anderson MD, Anderson GF, Bleecker GC, Rossi EC, Moss GS, Blood transfusion costs: a multicenter study,Transfusion 31, 2003, 318-323.

[18] Gillette BP, MaraditKremers H, Duncan CM et al, Economic impact of tranexamic acid in healthy patients undergoing primary total hip and knee arthroplasty,J Arthroplasty 28(Suppl), 2013, S137-S139. 